\title{
医用有机化学在线考试的探索与实践
}

刘坚华, 姚莉韵, 林琦, 杨若林 ${ }^{*}$

上海交通大学基础医学院，上海 200025

摘要: 通过总结本团队2020年春季学期对 “医用有机化学” 在线考试的考试方法、试题题型的设计, 试卷难度的调 控及防作弊措施的实施, 对有机化学在线考试优缺点进行了分析, 为今后有机化学课程开展在线考试提供参考。

关键词: 有机化学; 在线考试; 超星学习通

中图分类号: G64; O62

\section{Exploration and Practice of Online Examination of Medical Organic Chemistry}

Jianhua Liu, Liyun Yao, Qi Lin, Ruolin Yang *

Basic Medicine Faculty, Shanghai JiaoTong University, Shanghai 200025, China.

\begin{abstract}
This article analyzed the pros and cons of the online examination of organic chemistry. During the spring semester of 2020 , the teaching team carefully designed test papers and test methods to control the difficulty of test, thereby reducing the possibility of cheating, and successfully completing the online test. This article will provide a reference for the future online examination of organic chemistry courses.
\end{abstract}

Key Words: Organic chemistry; Online examination; Superstar learning platform

2020 年春季学期由于新冠肺炎疫情防控的需要, 各高校采用多种在线教学方式实践了教育部关 于 “停课不停教、停课不停学” 的要求 ${ }^{[1]}$ 。随着多数省份重大突发公共卫生事件响应级别降为三 级, 部分高校学生在 5 月中旬陆续返校, 教学活动逐渐回归正常。但仍有部分高校或部分年级的学 生未能在春季学期返校, 所有教学活动均需在线完成。如何保质保量完成在线期末考试就成为了线 上教学的一个挑战。

“医用有机化学” 是我校医学院为临床、口腔、儿科等专业一年级学生开设的必修课, 课程内 容涵盖基础有机化学理论及生物相关分子的结构与性质。该课程自开设 50 多年以来一直采用线下 闭卷考试的考核方式，近 5 年临床五年制专业的卷面不及格率在 5\%-20\%之间，八年制在 $10 \%-30 \%$ 之间(五年制学生由于要竞争免推直研名额, 平均不及格率通常低于八年制)。本学期由于我校大部 分医学院一年级学生不返校上课, 课程考核需在线完成。考虑到在线闭卷考试需要使用 zoom 远程 监考, 而本学期需要进行在线考试的学生超过 400 人, 在线监考对监考老师、监考设备、网络、学 生的考试设备、学生的摄像设备均有很高的要求, 同时有机化学课程很少测试仅凭记和背就能得分 的知识点, 因此我们选择了在线开卷、无人监考的方式进行本学期 “医用有机化学” 课程的考核。 
本学期我们的在线教学平台采用的是超星 “学习通”, 在线考试也在该平台完成。在教学过程 中我们曾发布了 12 份自测题, 题型包括单选、多选、书写结构式和完成反应式。在批阅自测题时我 们发现当有多道书写结构式和完成反应式的题目时, 由于需要拍照上传, 学生完成题目的时间差异 非常大, 同时网页加载速度较慢, 偶尔会出现图片无法显示的情况。为了更好地调控考试时间、避 免出现由于拍照设备和技术问题导致的图片清晰度较差、图片文件过大导致网页加载困难的问题, 同时, 考虑到北美医学院的入学考试 MCAT 全部为单选题, 通过合理设计试题能够满足考查学生有 机化学水平的目的, 在本次期末考试时我们放弃了有机化学考试中传统的书写结构式和完成反应式 的题目, 试题题型全部为选择题。

在正式考试前一周, 为了更加准确地了解学生的学习状况及他们使用的网络和设备情况, 我们 发布了 2 次模拟测试。第一次发布了 10 道题, 包括 1 道单选、9 道多选。10 道题目的 40 个选项全 部是图片, 包括波谱图、反应式、结构式和球棒模型图片, 这主要是为了测试学生使用的网络和设 备对试卷的加载能力。由于超星平台在试题输入和显示时会标明 “单选” 或 “多选” , 所以学生在 看到 “多选” 题时会知道至少有 2 个正确答案, 试题的整体难度会低于单、多混选型试题。教师标 定的题目难易程度是 “易” $40 \%$, “中” $40 \%$, “难” $20 \%$, 试题难度略高于以往考试。3 位授课教师 完成这 10 道题目的时间是 6-8 分钟, 给学生的答题时间设置为 12 分钟。测试结果表明, 波谱图片 由于像素较高, 无法在 App 端完整显示, 非常影响答题速度。另外, 从测试的及格率(30\%)和试卷提 交情况(多数学生没能在给定的时间完成测试)看, 学生普遍不适应多选题。因为单选题可以采用排 除法完成, 而多选题需要学生对所考核的知识点完全掌握才能答对。因此调整单、多选择题的比例 是调控在线考试试题难度的重要组成部分。

在第一次模拟测试后的第二天, 我们调整了题目的难度, 发布了第二次模拟测试。测试包括 15 道单选、5 道多选, 教师标定的题目难易程度是 “易” $45 \%$, “中” $55 \%$, “难” $5 \%$, 与最终考试的题 目难度相当。授课教师完成这些题目的时间为 10-12 分钟, 给学生设置的答题时间为 25 分钟。测试 结果表明此次题量合适, 五年制的试题难度较合适, 平均分为 59 分 (由于在考试前很多学生未开始 复习做题, 正确率较低), 区分度为 0.36 。八年制学生的平均分为 64 分, 区分度为 0.31 , 说明试题 难度需要微调。通过两次模拟测试, 我们确定了最终考试的题量为 100 道选择题, 五年制试题的单、 多选比例为 $3: 2$, 八年制试题的单、多选比例为 $1: 1$ 。经教学团队多位教师测试, 完成这些题目需 要 60-75 分钟, 预估学生 120 分钟可完成考试。

在设计试卷时教师标定为 “易” 的题目多数都是单选题, 仅考核一个知识点, 且题目较直观, 如题 1, 仅考查氨基酸等电点的概念, 学生只要掌握溶液 $\mathrm{pH}$ 与等电点之间的关系即可答对。标定为 “中” 的题目通常需要对知识点进行灵活应用, 如题 2。或者是考核多个知识点, 如题 3, 不仅考查 能发生 Diels-Alder 反应的共轭二烯的结构特点, 还考查了呋喃的化学性质。标定为 “难” 的题目不 仅考核多个知识点, 而且需要灵活运用, 或者有高于考试大纲基本要求的内容, 如题 4。这道题不仅 考查了 Hückel 芳香性的判断, 还考查了芳香杂环化合物。选项 C 是难度最大的一个选项, 需要对 Hückel 规则有深入的理解, 将化合物拆分为左右两半来考虑。另外, 在学习 Hückel 规则时, 学生往 往只关注 $\pi$ 电子的数目, 容易忽略 “共平面” 这个条件, 从而选择 $\mathrm{A}$ 选项。

题 1 天冬氨酸的 $\mathrm{pI}=2.77$, 它在 $\mathrm{pH}=10$ 的溶液中的存在形式为 $(\mathrm{B})$
(A) 正离子
(B) 负离子
(C) 两性离子
(D) 二肽

题 2 以甲苯为原料合成 5-硝基-2-澳-苯甲酸的步骤为( B )
(A) 先硝化、后溴化、再氧化
(B) 先溴化、后氧化、再硝化
(C) 先氧化、后硝化、再溴化
(D) 先溴化、后硝化、再氧化

题 3 下列化合物中能与乙烯发生 Diels-Alder 反应的是( A B C )
(A) 环戊二烯
(B) 1,3-丁二烯
(C) 呋喃
(D) 
题 4 以下化合物或中间体中具有 Hückel 芳香性的是( B C D )

(A)<smiles>C1=CC=CC=CC=C1</smiles>

(B)<smiles>C1=CCCC=C1</smiles>

(C)<smiles>C1=CC(=C2C=C2)C=C1</smiles>

(D)<smiles></smiles>

在无人监考的在线考试中如何防止出现作弊现象是至关重要的。此次考试学生是各自在家完成, 为了防止考试中出现学生通过网络互相询问答案的行为, 我们采取了如下措施: (1) 试题乱序、选 项乱序。这一措施可完全消除学生之间简单传递答案的行为。由于每位学生的考卷都是独一无二的, 如果要传递答案, 必需拍摄题目。由于超星考试平台的设置和电脑显示原因, App 端一次仅能显示 1 道题目, 网页端在复查页面一次也仅能显示 1-2 道题目。这大大增加了学生间问询答案的难度, 学生将很难通过拍题/截屏进而询问同学获取答案这种方式完成考试。(2) 题目量足够大。在线考试 答题期间, 无论是网页版还是 App 端一次均只能显示 1 道试题, 学生需要不停地按 “下一题” 按 钮, 完成同等试题量的时间比要纸质考卷长。根据 2 次模拟测试的结果, 学生完成试题的时间约为 教师的 2 倍。因此在 120 分钟内完成 100 道选择题对于学生来说时间不会很充裕(考虑到加载和提交 试卷的时间, 实际考试时长设置为 125 分钟), 优秀学生将没有时间 “帮助” 其他同学, 这也从另一 面杜绝了学生之间传递试题答案的可能。实测结果表明大部分学生在 118-124 分钟内提交了试卷, 小部分学生是在考试设置的时间截止时由系统强制提交。

期末考试前经征询学生意愿和调研学生家中设备、网络、电力情况, 有 3 位学生因家中停电维 修申请了缓考, 其余学生均参加了在线测试。403 位学生中除一位掉线 22 次, 一位掉线 6 次之外, 其余学生都顺利完成了考试。五年制的卷面平均分为 70 分, 不及格率 $24 \%$ 。八年制的卷面平均分为 69 分, 不及格率 $27 \%$ 。卷面成绩符合正态分布(图 1、图 2), 平均分略低于往年, 不及格率略高于往 年。这一方面说明试卷设计难易度合理, 防作弊措施是有效的, 另一方面也表明学生对在线考试的 适应度稍差(有机化学是该年级学生的第一门在线考试课程)。结合学生在线期间完成章节自测和作 业的情况, 此次考试成绩也说明学生居家在线学习的效果较在校学习差。

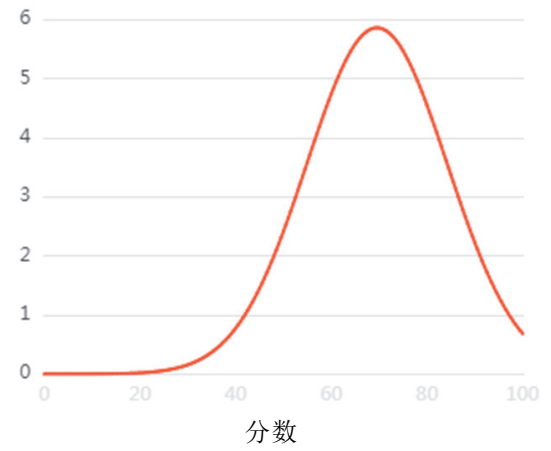

图 1 五年制成绩分布图

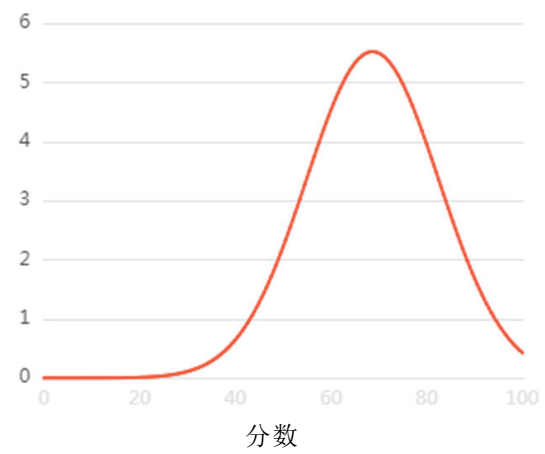

图 2 八年制成绩分布图

虽然本次考试是开卷考试, 但可以通过查询笔记获知正确答案的题目正确率并未达到 $100 \%$ 。如 要求标出甾体化合物骨架碳原子编号的题目, 正确率仅 $87 \%$ 。原因可能是部分学生没有提前准备好 需要查询的资料, 而答题时间较紧, 临时翻书和笔记犹如大海捞针, 无所适从。另外, 正如我们预 先估计的一样, 由于有机化学试题中能够通过直接查阅书和笔记获取正确答案的数量很少, 因此开 卷和闭卷考试在结果上并不会有本质的差异。

在对试题的难度系数进行分析时我们发现, 在八年制试卷中难度系数低于 0.3 的题目有 2 道。 题 5 是一道有关反应主产物的多选题, 难点是 $\beta$-羰基酸的脱羧。考试中有 $63 \%$ 的学生忽视了这个知 识点, 选择了答案 $\mathrm{C}$ 。这道题是教学安排中基础有机部分接近尾声的章节羧酸的考核知识点, 也是 我们为学生准备的课后习题中的题目。仅有不足 $30 \%$ 的学生能够做对该题说明大部分学生是考前突击 
学习, 尚来不及完成习题中靠后部分的题目。在以往线下教学中, 我们会通过每 3-4 章进行一次闭 卷章节测试的方式敦促学生学习, 但此次疫情期间由于学生是否返校、何时返校迟迟未定, 因此在 线教学期间仅发布了不计入期评成绩的自测和开卷作业, 很显然这样的方式对学生自主学习的敦促 力度不够。

题 5 以下反应的主要产物是( A D )<smiles>CC1CCC(O)C1C=CC1CC1CO</smiles>

(A)<smiles>O=C(O)C1CC1</smiles>

(B)<smiles>CC1CCC(O)C1C(=O)O</smiles>

(C)<smiles>CC1CCC(=O)C1C(=O)O</smiles>

(D)<smiles>CC1CCC(=O)C1</smiles>

此次在线考试的结果说明, 在合理调控试题量和试题难度的情况下, 可以通过在线方式完成有 机化学课程的考核, 考核结果与线下考试一样可以反映学生在课程学习中的问题。在线考试的优点 是当题目全部为选择题时, 可以由考试平台完成阅卷和试卷分析, 反馈非常及时, 因此可以在正式 考试前通过模拟测试确定最终的试题量和单、多选比例, 从而较好地调控试题难度。在试题量足够时, 开卷和闭卷考试的结果并没有明显差异。另外, 由于无需批阅试卷, 且很方便对题目的难度系数和 区分度进行统计, 如果将章节在线测试成绩计入期评, 一方面可以作为大班教学时敦促学生及时复 习的手段, 另一方面便于教师及时获知学生对知识点的掌握情况。而在线考试的缺点是由于题型限 制, 无法通过制备、简答等题型考查学生在有机化学课程学习之后综合运用知识的能力。在题目乱 序的情况下, 也无法通过给出题干, 将考核点分解为多道选择题的方式编写选择题, 对题目设计的 要求显著提高。同时, 由于题量增加, 虽然从教师的角度看每道题目的难度都不大, 但由于考核的 知识点增多, 对于部分习惯于考试前一周才开始学习的学生而言, 无形中增加了考核的难度。

全国高校的教师们通过一学期的实践, 已经体会到在线教学不仅仅是疫情之下不得已的教学替 代方案, 也可以是课堂教学的有益补充 ${ }^{[2,3]}$ 。同样, 在线考试或许也可以成为今后部分院校和专业课 程的考核方法之一。

\section{参 考 文 献}

[1] 朱亚先, 周立亚, 张树永, 郑兰䔉. 大学化学, 2020,35 (5), 283.

[2] 武全香, 惠新平. 大学化学, 2020, 35 (5), 48.

[3] 刘强, 彭娜. 大学化学, 2020,35 (5), 38. 